\title{
Penser la géologie dans la géographie militaire française (1871-1914)
}

\section{Philippe Boulanger}

\section{(2) OpenEdition}

\section{Journals}

Édition électronique

URL : https://journals.openedition.org/geohist/478

DOI : $10.4000 /$ geohist.478

ISSN : 2264-2617

Éditeur

Association française de la Revue de géographie historique

\section{Référence électronique}

Philippe Boulanger, «Penser la géologie dans la géographie militaire française (1871-1914) », Revue de géographie historique [En ligne], 14-15 | 2019, mis en ligne le 20 mai 2018, consulté le 12 juin 2021 URL : http://journals.openedition.org/geohist/478 ; DOI : https://doi.org/10.4000/geohist.478

Ce document a été généré automatiquement le 12 juin 2021.

\section{cc) $($ ) $\odot$}

$\mathrm{Ce}(\mathrm{tte})$ œuvre est mise à disposition selon les termes de la Licence Creative Commons Attribution-

Pas d'Utilisation Commerciale - Pas de Modification 4.0 International. 


\title{
Penser la géologie dans la géographie militaire française (1871-1914)
}

\author{
Philippe Boulanger
}

\section{Introduction}

1 La géologie est un domaine de pensée essentiel dans la géographie française à la fin du $\mathrm{XIX}^{\mathrm{e}}$ siècle et au début du XX'siècle. La culture militaire n'échappe pas à cette situation. L'étude de l'agencement des roches et des structures les affectant est une approche fondamentale, voire originelle, des débuts de la géographie militaire française. En effet, à partir des années 1870, la géographie militaire française s'impose comme un mouvement de pensée jusqu'aux années 1930, non sans retard par rapport à d'autres pays comme l'Espagne ou l'Allemagne. L'expression qui est diffusée par le professeur Théophile Lavallée à l'Ecole spéciale militaire de Saint-Cyr à partir de 1832, entre dans les dictionnaires militaires à la fin du siècle. Par exemple, le Nouveau dictionnaire militairepublié en 1892 la définit comme l'étude de la géographie d'un point de vue militaire en comprenant les mathématiques, les statistiques, la géographie politique et économique ainsi que la géographie physique (Anonyme, 1892). Il définit aussi la géologie révélant sa prépondérance pour l'activité militaire : « La géologie est l'étude de la terre à ses différents âges. Elle s'occupe de la forme et de la disposition des masses minérales qui constituent l'écorce solide du globe terrestre. Cette étude peut être très utile au général, à l'officier d'état-major et au topographe, parce qu'elle peut servir de base à des opérations de guerre, à des combinaisons stratégiques, à des plans de batailles, etc.» (Ibid.). Au cours de cette période de reconnaissance et de développement de la discipline géographique dans la culture militaire (écoles militaires, doctrine, études stratégiques), la géologie est une colonne vertébrale de tout raisonnement à des fins militaires. Elle propose, somme toute, une méthode scientifique bien commode et permet d'élaborer des hypothèses liées à la manœuvre de manière solide. En fait, l'un des paradoxes est que son succès contient les germes de sa remise en cause durant la Grande Guerre et de sa 
mutation au début des années 1920. Les théories déterministes se sont révélées inutiles dans la guerre de position à l'automne 1914, de sorte que la place de la géologie est repensée au profit de la géographie humaine. Comment la géologie s'impose comme un mode de raisonnement scientifique dans la géographie militaire française? Trois aspects sont abordés : la géologie à l'origine de la géographie militaire française, penser la géographie militaire par la géologie, les limites du raisonnement fondé sur la géologie.

\section{La géologie à l'origine de la géographie militaire}

\section{A. Une approche essentielle dans le développement des écoles latines et germaniques}

2 Comme l'a montré Hervé Coutau-Bégarie dans le Traité de stratégie, la géographie militaire est une approche de la géographie à des fins stratégiques qui naît au début du XIX ${ }^{e}$ siècle (Coutau-Bégarie, 1999). En tant que mouvement de pensée, différentes écoles se développent en Europe à partir des années 1810 et 1820. La raison est liée à la volonté de s'opposer aux armées napoléoniennes considérées comme des occupants que ce soit en Prusse, en Espagne ou dans les États de la péninsule italienne. L'analyse géographique devient ainsi un des moyens de contourner la puissance française en apportant un savoir stratégique et une connaissance du terrain. Les théoriciens de la stratégie qui ne sont pas géographes ont bien compris toute son importance pour les États-majors. Karl Von Clausewitz (1780-1831), acteur de la réorganisation de l'armée prussienne après la bataille d'Iéna de 1806, est nommé directeur de l'Académie de guerre de Berlin (1818-1830) où il valorise l'enseignement de la géographie. Son ouvrage De la guerre (1816-1831) accorde une place théorique mais importante aux territoires et à l'espace (montagnes, cours d'eau, etc.). Le stratégiste d'origine suisse Jomini (1779-1869), après avoir servi à l'État-major de Ney en 1805 puis du Tsar en 1813, rédige un Traité de grande tactique (1805) puis le Précis de l'art de la guerre (1837) qui accorde un chapitre à la prépondérance de la géographie militaire et de la statistique. À cette époque, ces deux auteurs témoignent d'un mouvement qui s'inscrit plus largement en faveur de la géographie militaire et conduit par des officiers qui présentent un raisonnement lié à l'espace.

3 Cette dynamique en faveur de la géographie militaire donne naissance à des écoles de pensée, d'abord dans les pays latins comme le royaume d'Espagne et dans les États italiens, puis dans les États germaniques comme la Prusse. Tous les écrits publiés dans les années 1810 à 1830 s'appuient, d'abord, sur la géographie physique, plus précisément sur la géologie. En Espagne, par exemple, le général Juan SanchezCisneros, dans Elementos de geographica fisica aplicada à la cienca de la guerra (1819), présente la manière d'exploiter la géologie pour la défense du territoire. Dans la péninsule italienne, la géographie militaire se développe pour la même raison tout en contribuant à la question de l'unité italienne. Dans les territoires germaniques, la Prusse devient une autre "Terre d'élection». Le Terrainlehre devient une science reconnue tandis qu'une section de cartographie est créée auprès de l'État-major dès 1816.

4 La Militar Geographie participe elle-aussi à réfléchir aux moyens défensifs du territoire, à la construction territoriale de l'unité allemande et à la Mitteleuropa. Entre autres 
exemples, le Maréchal Von Roon, dans Principes de géographie (1834) et dans Géographie militaire de l'Europe (1837), suit le même principe: envisager la prochaine guerre offensive et défensive à partir de la géologie et de la géographie physique. Dans l'Empire austro-hongrois, un Institut de géographie militaire est créé à Vienne tandis que plusieurs officiers enseignent et publient des traités de géographie militaire. Le colonel Von Rutdorffer élabore une Géographie militaire de l'Europe (1847) selon une approche descriptive et encyclopédique des connaissances de la Terre fondée sur la géologie, l'orographie et l'hydrographie. Dans l'Empire russe, en Roumanie, en Suisse, et au sein d'autres États européens à l'exception de la France et du Royaume-Uni, des mouvements de pensée contribuent à des productions scientifiques élaborées de manière inédite.

\section{B. Une approche encore marginale en France avant 1870}

5 Aux lendemains du Traité de Vienne de 1815, l'armée française est réorganisée de sorte qu'elle ne puisse plus être un obstacle à la stabilité du continent européen. Cette situation explique en grande partie le retard de la géographie militaire française jusqu'aux années 1870. Pourtant, l'expression de géographie militaire est déjà formulée dans le projet de la Convention d'une Agence des cartes au Dépôt de la guerre, en juin 1794, qui ne voit jamais le jour. Elle est reprise en 1832 par Théophile Lavallée dans ses cours à partir de 1832, puis son manuel intitulé Géographie physique, historique et militaire (Lavallée, 1832). Cet ouvrage connaît sept éditions jusqu'à sa mort en 1869 puis trois autres dans les années 1870 . Professeur de géographie et de statistique à l'Ecole spéciale militaire de Saint-Cyr, il est le premier véritable théoricien français de la géographie militaire. Son approche demeure académique en abordant une partie théorique sur les aspects physiques de la Terre et une partie régionale descriptive des bassins fluviaux dont l'étude s'achève par des considérations stratégiques.

6 La géologie apparaît centrale dans sa méthodologie en privilégiant, selon la conception académique de la géographie, la géographie physique selon les théories du XVIII ${ }^{\mathrm{e}}$ siècle. Le découpage régional s'appuie sur les bassins fluviaux qui permettent de comprendre les modes d'appropriation et d'organisation de l'espace par les sociétés. Théophile Lavallée conçoit même une méthode de géographie militaire en sept temps: la géographie physique, l'histoire des territoires, les bassins fluviaux, les côtes et les ports militaires, les sous-ensembles des bassins fluviaux, les frontières terrestres et le tableau stratégique (militaire). Toute l'originalité de cette méthode consiste à exposer le lien entre la géologie, et la géographie physique de manière générale, avec des considérations stratégiques inspirées des guerres napoléoniennes. Jusqu'aux années 1870, il apparaît le seul en France à s'y consacrer pleinement, révélant ainsi l'absence de réel courant de pensée mais aussi des retards structurels et culturels dans l'armée française qui éclatent au grand jour lors de la guerre franco-prussienne de 1870-1871.

\section{Une géographie militaire reconnue comme un savoir stratégique}

$7 \quad$ Au milieu du XIX ${ }^{\mathrm{e}}$ siècle, force est de constater que la géographie militaire est reconnue comme un savoir stratégique dans les pays latins et germaniques. Dans les académies militaires et dans les services géographiques militaires, elle est valorisée comme un pilier de la formation des officiers et de l'activité militaire. Cette reconnaissance révèle ainsi une prise de conscience de son utilité sur le plan institutionnel mais aussi une 
conception structurée. Son originalité porte justement sur cet aspect par rapport aux siècles antérieurs. Elle se structure en une méthode d'emploi et tente de proposer des hypothèses de réflexion à des fins militaires et politiques. Dans le développement de ces écoles de pensée de géographie militaire, la géologie constitue justement l'approche la plus scientifique qui est constamment recherchée. L'incidence de la nature des roches sur l'emploi des hommes et du matériel, sur la manœuvre et les axes de passage apparaît comme une finalité de la discipline.

8 En revanche, la géographie militaire française demeure trop peu développée pour être efficace en 1870. Si les bases académiques sont posées par Théophile Lavallée, elle demeure encore très marginale et peu inscrite encore dans la culture militaire. Les déficiences face à l'armée prussienne à l'été 1870 sont nombreuses en la matière. Que ce soit l'approvisionnement en cartes d'état-major du territoire français ou le niveau de connaissances des officiers, la géographie ne forme pas une priorité de l'art de la guerre. Aux lendemains de cette défaite en 1871, l'armée française en mesure toute l'importance.

9 Les établissements d'enseignement militaire sont alors restructurés. La discipline géographique est rendue obligatoire tandis que le niveau des connaissances exigées aux concours est élevé. Enfin, la création du Service géographique de l'armée en 1887, dont la vocation est d'abord de produire des cartes topographiques, contribue aussi au développement d'une réflexion sur l'usage du facteur géographique pour les armées. Il en résulte la naissance d'une école française de géographie militaire qui conduit à penser la discipline d'abord par la géographie physique.

\section{Penser la géographie militaire par la géologie}

\section{A. Une diversité de théories}

Ce mouvement de pensée de géographie militaire française est à la recherche d'une reconnaissance scientifique par l'élaboration d'une méthode d'analyse. Selon la tradition académique française, la géographie est d'abord physique. Il n'apparaît donc pas étonnant que les théories qui s'imposent jusqu'à la Grande Guerre s'appuient sur la géologie, l'hydrographie et la climatologie. En fait, seule la méthode par la géologie et l'hydrographie s'inscrit dans la durée et donne lieu à une diversité de théories.

11 La première est celle des Joints d'assises terrestres du colonel du génie Joseph Napoléon Fervel. Elle est exposée, d'abord, dans plusieurs articles du Spectateur militaire en 1870, intitulés "Études de géographie stratégique du Nord-Ouest de l'Europe », puis reprise dans l'ouvrage Études stratégiques du théatre de guerre entre Paris et Berlin en 1873 (Fervel, 1873). La méthode est inspirée de celle suivie par l'État-major allemand en s'appuyant sur la géologie et les régions naturelles et en écartant la théorie des bassins fluviaux ${ }^{1}$. Elle doit permettre d'identifier les espaces prévisibles de la prochaine guerre contre l'armée allemande. Les "joints d'assises terrestres" sont "les lieux les plus habituels des grandes batailles comme des grandes cités» (Fervel, 1870). Ils recouvrent étroitement "les bassins générateurs", caractérisés par leur unité géologique et ethnologique, et se juxtaposent aux « cases » des échiquiers de guerre. Ils peuvent se manifester, au-delà de leur dimension de lieu d'échanges historiques et de carrefour des communications, comme des lieux de rencontre culturelle. Fervel emploie l'expression de «barrières ethnologiques"pour désigner ces espaces de frontières culturelles, susceptibles 
d'apparaître comme des lieux d'affrontements armés entre deux peuples. Nombreux sont les exemples mis en évidence.

La région en amont de Magdebourg constitue l'un des champs de bataille prévisible de la prochaine guerre. En particulier, la plaine de Leipzig, "vers l'inévitable plaine de rassemblement qu'on a surnommée le carrefour des nations", est envisagée comme la base la plus rationnelle pour l'attaque du haut Elbe et de Berlin. Le Rhin, autre exemple significatif, sera l'enjeu et le théâtre d'un nouveau conflit, avant de devenir une fois encore une frontière naturelle. En somme, les opérations stratégiques sont soumises, de tout temps, aux "joints d'assises terrestres ». Cette approche, explique-t-il, justifie seule l'analyse des éléments physiques. La géographie militaire doit suivre des critères plus généraux, c'est-à-dire, "les contours des masses de même nature, les joints d'assises de la construction générale ». Elle s'appuie désormais sur les sillons des terrains, la géologie, la disposition des reliefs (l'orographie) et l'hydrographie.

La deuxième théorie est celle de la "géogénie » du commandant du génie Olivier Barré établie à la fin du XIX siècle. Sa conception est, en réalité, classique et reformule l'idée que la géologie est à la base de la géographie militaire qu'il enseigne à l'Ecole d'application du génie et de l'artillerie. Elle est diffusée entre 1897 et 1899 dans ses cours imprimés en sept fascicules qui portent sur l'Europe, la France et les colonies françaises: l'histoire morphogénique de l'Europe centrale, la France, la Belgique, la Hollande, la Suisse, l'Autriche-Hongrie, l'Italie du Nord et l'Allemagne, les colonies d'Afrique et l'Asie (Barré, 1897-1899). Elle est synthétisée dans la Géographie militaire etles nouvelles méthodes géographiques, Introduction à l'étude de l'Europe centrale et La France du Nord-Est, édité chez Berger-Levrault en 1899(Barré, 1899a). Toute son œuvre tend à asseoir la géographie physique dans la géographie militaire et à lui accorder une place prépondérante. Elle se veut scientifique par l'étude de bases solides que l'auteur situe dans la nature du sol. "La méthode géomorphogénique est la méthode nécessaire de l'enseignement de la géographie physique. Mais celle-ci est la base obligée de toute étude géographique spécialisée et en particulier de la géographie militaire " (Barré, 1899b, p. 78).

Cette méthode s'articule à partir de trois éléments distincts en rapport avec la géologie. Le premier est relatif à "l'architecture générale » de l'espace, c'est-à-dire qu'il s'intéresse d'abord aux éléments physiques homogènes du milieu. Cette étape doit permettre de décrire les grands ensembles et procéder à des subdivisions régionales. Le second renvoie à "la nature des matériaux", à la composition des roches et à leur incidence sur la conduite de la guerre. Le dernier porte sur " la sculpture du sol », accidents du terrain et obstacles naturels qui se présentent à une armée en mouvement. Ces trois éléments fixent les fondements de la géogénie, laquelle est complétée par d'autres caractères, liés aux voies de communication et aux positions défensives, qui restent cependant secondaires par rapport au déterminisme imposé par le milieu. L'étude du sol constitue l'essentiel de l'analyse d'un espace en géographie militaire, car la géographie se comprend avant tout par la géologie. Barré vient à regretter que les géographes français aient négligé les travaux des géologues, comme ceux de Elie de Beaumont. Selon lui, la configuration du sol établit les conditions de la marche des armées dans une région déterminée, mais son approche doit rester suffisamment synthétique pour aborder les phénomènes de masse et les mouvements de guerre. Lorsqu'il aborde la division des ensembles géologiques dans la France du Nord-Est, il distingue trois unités géographiques, «la Terre rhénane, la dépression de la Saône, la Région parisienne orientale ». 

première se compose d'une partie de la Terre rhénane et comprend les montagnes des Vosges et les plaines d'Alsace et du Palatinat rhénan, la Lorraine. La deuxième est composée de la haute vallée de la Saône, exposée directement à une invasion de l'armée allemande. Le troisième s'étend à toute la partie orientale de la région parisienne, de la Loire à Longwy. Ce théâtre d'opération présente la caractéristique de voir converger toutes les voies de communication provenant des deux premiers. Le principe de cette division régionale réside dans la distinction d'unités naturelles homogènes dont les périphéries sont des lieux de contact et de confrontation. Dans ces espaces en marge se situent les anciens et futurs champs de bataille selon l'avancée plus ou moins prononcée de l'adversaire. La démonstration stratégique consiste donc à comprendre d'abord ces unités naturelles dans leurs aspects géologiques et topographiques, puis à en déduire des hypothèses stratégiques allant jusqu'à proposer des aménagements de fortifications ou des plans d'opérations.

16 La troisième théorie est issue d'un auteur renommé parmi les géographes militaires puisqu'il s'agit de l'un des fondateurs de l'école française de géographie militaire. Gustave-Léon Niox (1840-1921), après une carrière brillante dans l'infanterie, est nommé professeur de géographie militaire dans la nouvelle Ecole supérieure de guerre en 1876. Il conserve ce poste jusqu'en 1895 après avoir été promu colonel en 1888 et officier général en 1893. Réputé pour son charisme et son talent oratoire, il forme ainsi plusieurs générations d'officiers français destinés à occuper des postes élevés au sein des États-majors. Il publie, surtout, ses cours de géographie militaire qui sont autant une encyclopédie en la matière : 7 ouvrages parus et réédités régulièrement entre 1876 et 1895. Le premier volume portant sur les Notions de géologie, de climatologie et d'ethnographie est édité en 1876 et réédité en 1878 et en 1880 , puis sous le titre de $L a$ France en 1882 jusqu'à 1893 ( $4^{e}$ édition) (Niox, 1876 ; 1882). Jusqu'en 1882, sa conception théorique de la géographie militaire est exposée dans ce premier volume. Elle s'appuie sur le même principe que l'étude de la géologie doit être le préalable à toute analyse de l'espace, idée qu'il modifie à partir des années 1880 pour accorder un raisonnement plus important à la géographie humaine. Selon Niox, la géologie détermine non seulement les conditions de développement d'une civilisation, mais aussi sa force militaire et sa capacité de s'imposer comme une grande puissance. Il admet que les opérations stratégiques sont commandées par les formes du relief, qui dépendent à leur tour de la composition géologique.

17 Ainsi, deux conditions principales fixeraient les hommes à s'établir dans une région et participeraient au développement d'un potentiel militaire. D'abord les richesses minéralogiques influencent "la manière d'être, de vivre, de travailler ». La "vitalité d'un pays, dont le développement est si intimement lié à sa prospérité agricole ou commerciale, dépend aussi en grande partie de la nature du sol» (Niox, 1878, p. 4). Il en déduit que la géologie intéresse la marche des armées. Sur un sol granitique, l'engagement des troupes reste possible par tout type de temps, contrairement au sol argileux qui se détrempe sous la pluie, rendant les routes impraticables, ou provoque une poussière pénétrante sous la sécheresse. La seconde condition s'explique par la fertilité des terroirs qui dépend de la constitution géologique des terrains et assure un potentiel de développement d'une société. Les « détails minéralogiques » constituent donc, selon lui, le premier critère d'analyse des nations. "La géologie vient en aide à l'histoire, permet des inductions utiles au militaire comme à l'homme politique et à l'administration» (op. cit., p. 5). 
Pour justifier cette théorie, Niox fait référence à la monarchie austro-hongroise divisée en deux grands États, différents par leurs mœurs, leur culture, leurs origines ethniques. Une ligne de démarcation s'appuyant sur des critères géologiques les sépare. Ainsi, en Hongrie, se situent les terrains composés de dépôts alluvionnaires occupés par les héritiers des envahisseurs magyares venant de l'Orient, de l'autre des terrains de soulèvement habités par des peuples de race allemande poussés par les invasions vers les contrées alpines considérées comme des refuges défensifs favorables. Ainsi, à l'origine de la géographie militaire, se découvre inévitablement le déterminisme de la géologie. "L'étude de la géologie d'un pays sera une initiative indispensable pour quiconque se propose d'en connaitre les conditions morales, économiques, stratégiques ».

Pour Niox comme pour Fervel et Barré, l'apport de la géologie à la géographie militaire doit constituer une base de réflexion scientifique. En 1878, par exemple, Niox reconnaît que les méthodes empiriques employées en géographie doivent être remplacées par une " méthode scientifique sérieuse ", c'est-à-dire par l'étude des roches car "l'ossature $d u$ sol, ses muscles, ses nerfs, sa vie pour ainsi dire, ses transformations qu'il faut comprendre et expliquer, et tout dans la nature ayant pour raison d'être une cause antérieure, c'est cette cause antérieure qu'il faut sans cesse remonter » (op. cit.,pp. XI-XII).

\section{B. Une conception de la géographie militaire fondée sur la géologie}

Ces trois théories traduisent, en réalité, un contexte de pensée qui atteste de la prépondérance de la géographie physique. Elles influencent toutes bien d'autres auteurs en géographie militaire jusqu'à la Grande Guerre. Leur portée est donc considérable pour plusieurs générations d'officiers et suscitent des débats entre eux. Elles présentent aussi le point commun de procéder à l'étude de la géologie pour comprendre le terrain. Or le terrain est la condition première de la conduite de la manœuvre. Le terrain commande l'action comme l'écrivent nombre de théoriciens de la stratégie ou de la tactique au $\mathrm{XX}^{\mathrm{e}}$ siècle. Certains font aussi référence à « la tyrannie du terrain ». Comment est ainsi envisagée la relation entre la géologie liée au terrain et l'art militaire?

20 Tout d'abord, un premier niveau de réflexion se rencontre dans des considérations générales à travers l'ensemble des études. Le terrain est lié à la tactique, puis à des considérations stratégiques selon l'échelle géographique envisagée. Il importe de savoir pour le militaire la nature des sols (imperméables ou perméables), l'organisation du relief (axes des vallées et des sols), la composition de la végétation (l'obstacle formé par la forêt dont la nature dépend en partie des couches géologiques), la climatologie (vent, température). Ensuite, l'étude de la géologie conduit à analyser le terrain selon sa valeur défensive. Les accidents du sol peuvent offrir des avantages pour arrêter une avance ennemie, pour élaborer une fortification de campagne par exemple. Enfin, cette étude conduit à comprendre comment les couches géologiques déterminent l'occupation humaine et l'activité économique. Celles-ci influencent, pensent les géographes militaires, la densité de population, les capacités de production agricole, les ressources industrielles et commerciales. Ces trois niveaux de réflexion sont récurrents et révèlent une pensée déterministe.

21 À un autre niveau de réflexion, les composants géologiques constituent un des trois piliers de la géographie militaire avec la topographie et le climat. Ils exercent une influence dans la guerre de mouvement et de positions. Les sols sont ainsi classés selon 
leurs propriétés et leurs valeurs militaires s'ils sont meubles, sablonneux ou lourds. La nature des roches définit un cadre d'intervention plus ou moins facilité. La structure des sols conditionne les formes d'interventions militaires. La résistance et la perméabilité des roches qui varient selon les régions conduisent à des conditions d'action préétablies. Des sols peu résistants et perméables sont favorables au stationnement des troupes et au creusement de tranchées et d'abris. Les inondations dans les cantonnements souterrains ou à la surface se font rares sinon inexistantes. Les infections transmises par l'eau stagnante apparaissent aussi plus rares.

La géologie informe ainsi sur les conditions extérieures au combat, celles que les combattants rencontrent avant, pendant et après la bataille. Elle renseigne encore sur les matériaux disponibles pour l'organisation des défenses. Les roches sont généralement nécessaires et utilisées pour la construction de bâtiments et d'abris. Elles constituent une véritable ressource à exploiter à condition de disposer de personnel instruit et compétent. Enfin, elle permet d'envisager les conditions d'emploi du matériel, des systèmes d'armes et des hommes. Tout l'intérêt de la géographie militaire porte sur la maitrise de la connaissance du terrain pour le militaire durant cette période. Parallèlement, il apparaît une autre dimension du raisonnement géographique. La géologie sert aussi à mieux préparer et conduire la guerre de mouvement.

\section{La naissance de la « géologie militaire » pour la guerre de mouvement}

Alors que la guerre de position et la fortification de campagne ne sont guère des principes dominants de la pensée militaire à la fin du XIX siècle et au début du XX siècle, la guerre de mouvement est considérée par l'État-major général comme inévitable et la seule doctrine possible. Il apparait donc cohérent que les géographes militaires s'intéressent à l'usage des couches géologiques pour comprendre les voies d'invasion, à l'influence des sols pour la mobilité et le transport. Les considérations apportées à des fins tactiques ou stratégiques apparaissent donc primordiales pour connaître les possibilités de mouvements sur les axes d'invasions, d'emploi des armes et des hommes sur de grands espaces. Il en résulte l'émergence de l'expression de "géologie militaire " dans plusieurs ouvrages à la fin du XIX ${ }^{e}$ siècle, notamment ceux de Charles Clerc qui semble avoir été l'inventeur de cette expression en France. Charles Clerc défend cette conception de la géographie militaire dans Géologie militaire, les Alpes françaises (1882) et dans Géologie militaire, le Jura (1888) (Clerc, 1882 ; 1888).

Pour les géographes militaires, comme Laurent Pichat dans Géographie militaire du bassin $d u$ Rhin (1876), la géologie militaire joue un rôle important dans la guerre de mouvement (Pichat, 1876). Les roches déterminent des possibilités de déplacement élémentaires et conduisent les armées à faire face à des difficultés d'équipement plus ou moins importantes selon les cas. Sur les roches granitiques, la mobilité reste satisfaisante en toute saison et ne se heurte à aucune difficulté majeure. Sur les roches calcaires, les chemins deviennent plus difficiles, par la présence de la poussière sur le matériel et les hommes en été, par les pluies qui les rendent glissants. Sur les sols de craie pure ou légèrement marneux, peu de difficultés sont rencontrées. Il est reconnu que les pays crayeux facilitent les déplacements de masse et la coordination de tous les éléments qui composent les armées modernes comme le Vermandois et la vallée de 
l'Oise. Sur les sols argileux et marneux, les conditions de mobilité se durcissent lorsque la pluie ouvre de larges chenaux boueux. Les armées sont alors ralenties sinon alourdies par la boue. Cette catégorisation des roches par rapport à la mobilité fait partie de la conception de la géographie militaire jusqu'aux années 1930. Elle apparaît essentielle même dans la guerre de position durant la Grande Guerre alors qu'elle était envisagée pour la guerre de mouvement.

Dans Les conditions géographiques de la Grande Guerre, thèse de doctorat soutenue en Sorbonne en 1923, le géographe militaire Robert Villate s'en inspire et l'illustre de nombreux exemples tout en apportant des possibilités d'aménagement (Villate, 1925). Il considère que sur les sols argileux en temps de pluie il faut soit sortir de la zone comprenant ces sols déshérités, soit apporter suffisamment de matériaux pour ne plus en dépendre. Dans tous les cas, des sols marneux et argileux sont sources d'épuisement pour les soldats. En décembre 1914, le $21^{\mathrm{e}}$ corps d'armée lance une offensive sur l'éperon de Notre-Dame-de-Lorette au Nord d'Arras. Il échoue en raison de la nature du sol, argileux et imperméable. Les soldats peinent à sortir de la tranchée, glissent sur le sol et s'exposent ainsi maladroitement aux tirs de l'ennemi protégé.

Enfin, la conception de la géologie à des fins militaires présente aussi son intérêt pour l'emploi des armes, essentiellement l'infanterie, la cavalerie et l'artillerie avant 1914. Selon ces armes, la nature des roches n'exerce pas autant d'influence. Si les dispositions déjà citées sont considérées pour l'infanterie, la nature des sols occupe une place essentielle pour l'artillerie. Dans les roches meubles et imperméables, les obus s'enfoncent sans éclater et les tirs d'artillerie perdent une partie de leur efficacité. Dans le sol humide, les pièces d'artillerie se désappointent, réclamant un replacement systématique, réduisant de fait la cadence des tirs. Ces situations se rencontrent durant la Grande Guerre. L'artillerie française, dans la Woëvre en avril 1915, se heurte ainsi à ce problème. Les soldats français, lancés à l'attaque de la première ligne allemande, découvrent des réseaux de fils de barbelés et une défense restée intacte (Ibid., p. 27). Pour y faire face, de nouveaux procédés sont généralisés par la suite sur le front. Sur les sols argileux, des types d'obus sont conçus pour éclater au niveau du sol tandis que les armées apprennent à classer les types de terrains selon leurs propriétés naturelles et militaires. Cette catégorisation des couches géologiques est même

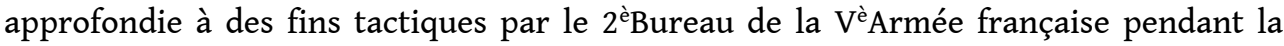
guerre 1914-1918. Comme le montre le tableau 1, celui-ci identifie les qualités des roches avec l'emploi des armes témoignant d'une continuité des réflexions d'avantguerre en tenant compte des expériences des offensives successives dans la région de Reims. 
Document 1 : L'influence des roches géologiques sur l'emploi des armes

\begin{tabular}{|c|c|}
\hline Argile plastique & $\begin{array}{l}\text { Humidité préservée, praticable à l'infanterie, difficulté possible pour } \\
\text { l'artillerie de campagne et l'infanterie après les pluies }\end{array}$ \\
\hline Sables argileux & $\begin{array}{l}\text { Toujours imbibés d'eau sous la forme de limon des plateaux ou sur les } \\
\text { pentes des collines, terrain lourd peu praticable sous la pluie. }\end{array}$ \\
\hline Sables purs & $\begin{array}{l}\text { Perméables, assèchement rapide, praticables en tout temps et à toutes } \\
\text { armes. }\end{array}$ \\
\hline $\begin{array}{l}\text { Craie argileuse ou } \\
\text { marneuse }\end{array}$ & $\begin{array}{l}\text { Humide par la présence d'un niveau aquifere, progression rapide gênée } \\
\text { par un sol glissant, voire difficile pour l'artillerie sur les pentes. }\end{array}$ \\
\hline $\begin{array}{l}\text { La craie pure ou légè- } \\
\text { rement marneuse }\end{array}$ & $\begin{array}{l}\text { Sol résistant, excellent pour toutes les armes, faible impact de la pluie } \\
\text { malgré la présence d'une boue légèrement argileuse, terrain solide mais } \\
\text { glissant. }\end{array}$ \\
\hline Calcaire grossier & $\begin{array}{l}\text { Sol résistant et dur, aucun impact de la pluie, praticable pour toutes les } \\
\text { armes. }\end{array}$ \\
\hline
\end{tabular}

Source : 2 è Bureau de la Vème Armée française pendant la guerre 1914-1918 (cité dans Villate, 1925, p. 29)

En somme, la géographie militaire française entre 1871 et 1914 se pense par la géologie. Force est de constater qu'elle est, d'abord, envisagée dans le cadre d'une géographie terrestre. La géographie maritime et littorale apparaît marginale tandis que la géographie militaire aérienne n'apparaît qu'à partir de l'Entre-deux-guerres. Ensuite, l'apport de la géologie dans le raisonnement militaire se conçoit à partir de l'idée de mouvement. Il est pris en compte pour l'aménagement du terrain surtout à partir de la Grande Guerre dont la première instruction doctrinale ne date que de 1917. Ce qui importe, avant 1914, est de connaitre les possibilités de déplacement des armées sur des axes d'invasion et des théâtres de guerre déjà identifiés. Enfin, la réflexion géographique militaire est centrée sur le continent européen et pour un milieu tempéré ou méditerranéen. Il apparaît peu de connaissances ou d'intérêts stratégiques pour les autres continents. Il est vrai, aussi, que cette conception de la géographie militaire, dépassée à l'entrée en guerre en septembre 1914, présente plusieurs limites.

\section{Les limites d'une géologie militaire opérationnelle}

\section{A. Une conception déterministe de la géographie}

L'école française de géographie militaire est l'une des plus brillantes en Europe, par la diversité des études régionales comme par le nombre d'auteurs (près d'une centaine). Mais elle présente une conception déterministe de la géographie alors que la géographie universitaire s'en détache justement à partir de la fin du XIX ${ }^{\mathrm{e}}$ siècle. Jusqu'en 1914, la théorie des joints d'assises terrestres, dont l'expression ne connaît pas de succès après son invention par Fervel, est reprise par bien d'autres auteurs. Le principe a le mérite d'être simple et clair. Tous les éléments d'une société (la population, les ressources, etc.) sont imprimés dans le sol. L'histoire des nations s'explique par la nature du sol et la similitude des terrains constitue la véritable unité de la géographie. Le joint d'assise terrestre se caractérise alors comme le lieu de 
rencontre de deux types de terrain, donc de deux peuples opposés qui se sont affirmés à la surface de roches homogènes. Cet espace de rencontre est avant tout un espace de heurt, de rivalité et de confrontation. Les guerres, explique son auteur, le colonel Fervel, se déroulent à l'emplacement de ces lieux de transition et peuvent être prévues en conséquence.

Cette théorie des joints d'assises terrestres inspire plusieurs travaux avec des variantes selon les cas. Braeckman et Ducarne, dans Géographie militaire de l'Allemagne, de la Hollande, de la France et de la Belgique (1878), débutent leurs analyses régionales par la géologie et en déduisent, ensuite, les particularités. Chaque unité géologique forme alors un théâtre de guerre qui est ensuite étudié dans ses aspects militaires, comme les fortifications. Le successeur de Théophile Lavallée à l'Ecole spéciale militaire de SaintCyr, Bureau, dans la Géographie physique, historique et militaire (1882), considère que la géographie militaire se définit comme l'étude du sol appliquée à l'art de la guerre. Son analyse conduit à comprendre les lignes de fracture, les accidents topographiques qui ont déterminé l'histoire des États. Les grandes vallées apparaissent comme des zones de contact évidentes entre deux joints d'assises terrestres. Lorsqu'il évoque la géographie militaire de la "région française ", il met en évidence les bassins fluviaux comme des entités naturelles homogènes et des lieux de contact entre peuples. Il considère, par exemple, que le bassin du Rhin supérieur, entre le Pô, le Danube et le Rhin moyen, constitue le centre des trois grands théâtres de guerre de l'Europe occidentale. Il fait de la Suisse une «position stratégique des plus remarquables, éminemment favorable à l'offensive, à cause des débouchés qu'elle procure dans chacun de ces bassins " (op. cit., p. 132).

Le bassin du Rhin supérieur s'ouvre vers la vallée du Danube par la ligne BâleSchaffouse, vers le Tyrol par Feldkirch, vers le coeur de l'Italie septentrionale par le Splugen ou le Saint-Gothard. Quant aux frontières du Nord-Nord-Est, les limites ne s'alignent pas sur celles des unités physiques, d'où la succession de guerres entre les États depuis le XVII ${ }^{\mathrm{e}}$ siècle. Toutes les démonstrations stratégiques de Bureau s'appuient sur la théorie des lieux de contact, des joints d'assises terrestres, de la correspondance entre les grandes unités naturelles et notamment fluviales.

31 Toute la conception de la géographie militaire renvoie ainsi au primat de la géologie, c'est-à-dire à l'idée que la composition des roches joue un effet déterminant dans la conduite des opérations. La question que pose l'ensemble des géographes militaires dans leurs travaux respectifs est celle de l'adaptation de la troupe au milieu naturel. Les raisonnements préconçus font partie des connaissances géographiques générales dont doivent disposer les officiers. La finalité de la géographie militaire réside donc dans la préparation de ces éventualités contraignant la conduite des opérations, soit par l'étude des cartes géologiques, soit par l'apprentissage du terrain. Charles Clerc accorde ainsi une place essentielle à la géologie dans ses deux études sur les Alpes et le Jura. Il s'annonce d'ailleurs le fondateur d'une méthode nouvelle qu'il nomme la "géologie militaire »et qui tend à valoriser l'étude des roches et du relief à des fins militaires. Dans les Alpes françaises, études de géologie militaire (1882), il développe plusieurs théories de la défense des Alpes contre l'Allemagne et l'Italie après avoir traité de l'origine et la formation des Alpes (orogénique, configuration, hydrologie, climatologie), la géologie des régions de Savoie, Alpes valdôtaines, Dauphiné et Alpes vaudoises. La géologie militaire se veut d'abord générale et largement inspirée de la géologie pratiquée alors par les membres de la Société de géographie dont il est membre. Le fait militaire n'intervient finalement que sous la forme de conclusion. Dans Le Jura, études de géologie 
militaire (1888), il privilégie, à partir de la géologie surtout l'étude des axes de passages et les éventuels mouvements d'un adversaire italien.

Cette méthode reposant sur la géologie présente toutefois des limites. D’abord, la complexité relative de la géologie rebute des auteurs comme des lecteurs potentiels, limitant ainsi le foisonnement intellectuel de la fin du siècle. La géologie apparait être le gage d'une géographie sélective et de qualité. En outre, cette méthode fixe un cadre de réflexion rigide, laissant peu de place à de nouvelles hypothèses. Les théâtres de guerre se rencontrent toujours aux mêmes lieux, certaines régions apparaissent toujours difficilement praticables comme le souligne, en 1892, le Nouveau dictionnaire militaire: "La géologie indique les joints des assises qui composent chaque élément de la surface terrestre, et, par suite, les crêtes ou accidents de terrain susceptibles de constituer de bonnes lignes de défense. Ces joints correspondent à des accidents de terrain ayant une grande valeur au point de vue tactique et stratégique; aussi l'histoire militaire permet de constater que les grandes batailles se sont livrées très souvent des localités situées aux joints d'assises terrestres. On peut donc dire que si ce n'est pas la géologie elle-même qui influe sur les combinaisons de la guerre, elle n'intervient pourtant indirectement par le relief, par le modelé de la croûte terrestre qu'elle implique. A ce point de vue, elle est le complément nécessaire de la géographie militaire. Un simple coup d'œil jeté sur une bonne carte géologique fera immédiatement connaître les points importants des lignes naturelles de défense ».

\section{B. Une ouverture limitée vers la géographie universitaire}

Une autre limite se distingue dans l'élaboration d'une géographie militaire qui se veut opérationnelle et concrète pour le militaire. Celle-ci présente aussi, d'après les sources disponibles, un certain repli sur elle-même, fort éloigné des mutations de la géographie universitaire. Les progrès de la géographie vidalienne ne sont pas connus avant la Grande Guerre. Sous l'impulsion de Paul Vidal de la Blache (1845-1918), de nouvelles conceptions se développent, mettant en valeur le rapport entre l'homme et le milieu, les données de la récente géographie humaine où les concepts de régions naturelles, de genres de vie, de civilisation industrielle et de circulation sont approfondis ${ }^{2}$. Le " possibilisme » qui suppose que les conditions physiques de l'espace, qui influencent les sociétés dans leur manière de s'organiser, peuvent aussi être modelées par celles-ci, s'impose au tournant du siècle à la Sorbonne et dans les enseignements de la géographie française dans les facultés. Il tend à remplacer le déterminisme géographique et crée une mutation importante en termes de méthodologie et de raisonnement.

Or celle-ci ne semble pas avoir été connue des géographes militaires à cette époque. Les documentations consultées par les géographes militaires, outre les cartes géologiques et topographiques, s'appuient surtout sur les travaux des géographes allemands du $\mathrm{XIX}^{\mathrm{e}}$ siècle, les guides de voyage, parfois la Géographie universelle d'Elisée Reclus ou les ouvrages de Levasseur, professeur de géographie économique et de statistiques au Collège de France à partir de 1868. Les géographes militaires ont peu accès aux récents travaux de recherche de l'école vidalienne ou aux numéros de la Revue de géographiede la Société de géographie de Paris (laquelle comprend des officiers de l'armée coloniale mais très peu d'officiers métropolitains), le Bulletin de géographie historique et descriptive ou la nouvelle revue des Annales de géographie créée en 1891. Enfin, ils demeurent attachés à la théorie des bassins fluviaux d'Elie de Beaumont ou de Philippe 
Buache datant du XVIII ${ }^{e}$ siècle. Celle-ci apporte un cadre commode sur le plan scientifique pour lequel il est peu envisagé de transformation.

Parallèlement, les géographes militaires, tous officiers de carrière en poste dans des sites militaires à Paris comme en province, n'ont guère le temps ou les moyens de fréquenter les cours de géographie à l'Université. Ils sont presque tous des professeurs de géographie dans des écoles militaires, dont l'instruction s'est effectuée souvent avant la guerre de 1870-1871, autrement dit à une époque où la géographie française est encore largement influencée par la géographie physique et historique. A l'exception de Charles Clerc, qui est membre de la Société de géographie et non professeur, tous ne côtoient pas le milieu universitaire, qui ne comprend d'ailleurs qu'un faible nombre de professeurs en France. La géographie militaire se veut avant tout une géographie appliquée et moins un exercice intellectuel destiné à l'obtention d'un diplôme. Il faut attendre la création de la Commission de géographie au sein du Service de géographie de l'armée, en octobre 1914, pour que les géographes universitaires et les géographes militaires travaillent ensemble sur des notices régionales en Europe intéressant les armées françaises.

Il en résulte un désintérêt pour la géographie universitaire qui apparaît trop moderne, peu de relations avec la communauté des géographes académiques et une Ecole française de géographie militaire isolée qui ne se renouvelle pas à la suite des nouvelles formes de conflits qui apparaissent en Asie orientale ou dans les Balkans au début du $\mathrm{XX}^{\mathrm{e}}$ siècle. A la veille de la Grande Guerre, les théories de géographie militaire se révèlent inopérantes et ont perdu de leur rayonnement par rapport à la fin du XIX ${ }^{e}$ siècle.

\section{Une conception de la géologie réservée au milieu militaire}

La géographie militaire française avant 1914 présente cette particularité d'être destinée au seul milieu militaire. Sa diffusion est destinée à un public averti et sensibilisé à des questions stratégiques en vue de préparer la prochaine guerre contre l'Allemagne. En quête de reconnaissance au sein de l'institution militaire, elle s'impose comme un outil pragmatique et concret et articule ses démonstrations à partir de problématiques stratégiques et tactiques. Dans La France par rapport à l'Allemagne (1884), l'auteur anonyme, probablement le colonel Rémy, a conscience de s'adresser à des lecteurs d'abord militaires. «Le travail que nous présentons ici, particulièrement au public militaire, a un objet spécial et restreint qu'il importe de bien définir. C'est une étude, au point de vue stratégique, du territoire de la France par rapport à l'Allemagne (...)» (Anonyme, 1884, p. V). Certains officiers issus des écoles militaires poursuivent cette réflexion et l'enseignent à leur tour devant une nouvelle génération d'élèves-officiers. Bureau, ancien élève de l'Ecole militaire de Saint-Cyr, succède à Théophile Lavallée à la fin des années 1860. Cette diffusion se veut donc réservée aux élites militaires. Elle ne concerne pas un public large, contrairement à la géographie universitaire qui connaît un plus grand rayonnement au sein de la société française.

Les théories précitées, reposant sur l'usage de la géologie à des fins militaires, limitent par leur complexité sa diffusion au-delà de la communauté militaire. Elles exposent la finalité appliquée de la géographie militaire. Celle-ci ne se veut pas uniquement un exercice purement intellectuel mais une discipline concrète, répondant à un besoin d'ordre tactique et stratégique. L'ouvrage de Luzeux sur Le Morvan, étude physique, 
historique et militaire, publié vraisemblablement dans les années 1880, tend à faire comprendre le rôle stratégique que pourrait jouer le Morvan en cas de guerre. Cette région serait alors une vaste place militaire, une zone de refuge pour effectuer une contre-offensive. Cet essai de géographie militaire répond au besoin de valoriser une région stratégique sous-estimée et revêt un caractère avant tout pratique qui n'intéresse qu'un public de lecteurs avertis.Il témoigne également d'une conception plus problématisée et moins encyclopédique de la discipline qui n'en demeure pas moins réservée à la communauté militaire et éloignée des enjeux de la géographie universitaire.

Pour ces différentes raisons, la géographie militaire renforcée des théories dites scientifiques s'appuyant sur la géologie se limite à un public restreint qui ne parvient déjà plus à convaincre les officiers d'Etat-major à la veille de la guerre. La modernisation des systèmes d'armes, comme l'artillerie ou l'apparition de l'usage de l'avion pour la reconnaissance, ne peut être prise en compte et suscite un autre intérêt au détriment de la discipline géographique. Si la carte d'Etat-major demeure un outil permanent des officiers, la théorie des Joints d'assises terrestres semble avoir été oubliée en raison de sa nature déterministe au sein du milieu militaire.

Entre 1871 et 1914, l'essor de la géographie militaire française est étroitement lié à une conception traditionnelle de la géographie, reposant essentiellement sur sa dimension physique. Cet essor s'inspire de modèles européens, principalement latins et germaniques, qui avaient conçus, au début du XIX ${ }^{\mathrm{e}}$ siècle, une discipline géographique opérationnelle contre l'armée napoléonienne, puis comme un savoir stratégique pour tout officier. La défaite française face à la Prusse en 1870-1871 suscite de profondes mutations en faveur de son développement dans les décennies suivantes, dans le cadre de la reconstruction de l'appareil militaire. Une véritable école de pensée française émerge et valorise le raisonnement géographique à des fins militaires.

Or ce raisonnement s'appuie en grande partie sur la géographie physique et sur la géologie que le géographe militaire Charles Clerc invite à renommer, en 1882, par l'expression de Géologie militaire. Si les théories géographiques militaires entretiennent surtout une forme de déterminisme, il n'en demeure pas moins qu'elles ont suscité un certain engouement à la fin du XIX ${ }^{\mathrm{e}}$ siècle et au début du XXe siècle. Elles participent à créer un mouvement de pensée qui connaît son âge d'or durant cette période. A l'entrée en guerre en 1914, elles apparaissent inopérantes sur un plan stratégique. Mais l'usage de la géologie à des fins militaires demeure de premier intérêt dans la guerre de position. La réflexion géographique militaire s'engage alors dans une autre phase de son développement. Tout en conservant un intérêt pour la géographie physique dans son ensemble, elle intègre davantage la géographie humaine à des fins militaires.

\section{BIBLIOGRAPHIE}

Anonyme, 1884, La France par rapport à l'Allemagne, étude de géographie militaire, Bruxelles, Marquardt, $375 \mathrm{p}$. 
Anonyme, 1892, Nouveau dictionnaire militaire par un comité d'officiers de toutes armes, Paris, Baudoin, $854 \mathrm{p}$.

Barré O., 1897-1899, Cours de géographie, croquis de géographie, Fontainebleau, Ecole d'application.

Barré O., 1899a, La géographie militaire et les nouvelles méthodes géographiques, Introduction à l'Etude de l'Europe centrale et la France du Nord-Est, Paris, Berger-Levrault, 80 et 122 p.

Barré O., 1899b, Cours de géographie, croquis de géographie, Introduction à l'Etude de l'Europe centrale, Paris, Berger Levrault, p. 78.

Clerc C., 1882, Les Alpes françaises, études de géologie militaire, Paris, Lib. Berger-Levrault, 224 p.

Clerc C., 1888, Le Jura, études de géologie militaire, Paris, Berger-Levrault, 215 p.

Coutau-Bégarie H., 1999 ( $2^{e}$ éd.), Traité de stratégie, Paris, Economica, 1005 p.

Fervel, Colonel, 1870, «Etudes de géographie stratégique sur le Nord-Ouest de l'Europe », Le spectateur militaire, p. 21.

Fervel, Colonel, 1873 ( $2^{\mathrm{e}}$ éd.), « Etudes de géographie stratégique », Le Spectateur militaire, juin 1870, pp. 312-332, juillet-septembre 1870, pp. 5-27,

Fervel, Colonel, 1873, Etudes stratégiques sur le théâtre de guerre entre Paris et Berlin, Paris, Dumaine, $152 \mathrm{p}$.

Lavallée Th., 1832, 1836, 1853 (4e éd.), Géographie physique, historique et militaire, Metz, Roussel Jeune, $612 \mathrm{p}$.

Niox G.-L., 1876 (1ère éd.), Notions de géologie, de climatologie et d'ethnographie, Paris, Dumaine, 1876, $1878,1880,191 \mathrm{p}$.

Niox G.-L., 1882 (1ère éd.), La France, Paris, Baudoin et Delagrave, 1882 à 1893, 4eme édition, 440 p. Niox, Commandant, 1878, Géographie militaire, Introduction, Paris, Dumaine, p. 4.

Pichat L., 1876, Géographie militaire du bassin du Rhin, Paris, Delagrave, 304 p.

Villate R., 1925, Les conditions géographiques de la guerre, étude de géographie militaire sur le front français de 1914 à 1918, Paris, Payot, 325 p.

\section{NOTES}

1. Il en appelle à renoncer à la méthode des bassins hydrographiques, encore suivie dans la géographie militaire de Lavallée. Ces bassins fluviaux ne représentent que des "rayures accidentelles", des «gouttières errantes ", une "énumération d'accidents sans suite » qui désespèrent la mémoire.

2. De 1895 à 1905, Vidal de la Blache, professeur successivement à l'école normale supérieure, aux universités de Nancy et de la Sorbonne à Paris, pose les bases d'une nouvelle géographie qui s'organise en une école de pensée. Il crée les Annales de géographie en 1896, encadre de nombreuses thèses, assurant ainsi la pérennité de sa pensée. À partir de 1905, l'école de géographie française est définitivement établie et reconnue dans le monde entier, grâce aux travaux des disciples de Blache, notamment de ceux de Raoul Blanchard, Jules Sion, Camille Vallaux, Jean Bruhnes, Emmanuel de Martonne, Albert Demangeon. Il est à noter que les géographes universitaires ne s'intéressent pas, par ailleurs, à la géographie militaire. 


\section{RÉSUMÉS}

La géographie constitue un savoir stratégique, opérationnel et tactique qui est longtemps resté empirique. Elle tend à se construire plus précisément à partir du XIX ${ }^{\mathrm{e}}$ siècle en Europe, notamment en s'appuyant sur la géologie. En France, la géographie militaire forme un véritable courant de pensée après la défaite face à la Prusse en 1870 , dont l'intérêt est de se préparer à la prochaine guerre face à l'Allemagne. Ses théoriciens tendent à concevoir des méthodes scientifiques et des finalités qui doivent la rendre opérationnelle. La géologie est ainsi considérée comme l'approche fondamentale, mais pas unique, de la manière de penser le territoire à des fins militaires jusqu'à la Grande Guerre. Des théories élaborées sont diffusées dans de multiples études comme enseignées dans les écoles militaires. Comment le primat de la géologie s'est-il imposé dans la géographie militaire française jusqu'en 1914 ?

Geography is strategic, operational and tactical knowledge that has long been empirical. It tends to be built more precisely from the 19th century in Europe, in particular by relying on geology. In France, military geography formed a real stream of thought after the defeat of Prussia in 1870, whose interest was to prepare for the next war against Germany. His theorists tend to design scientific methods and purposes that should make it operational. Geology is thus seen as the fundamental, but not unique, approach to thinking about the territory for military purposes until the Great War. Developed theories are disseminated in multiple studies as taught in military schools. How did the primacy of geology prevail in French military geography until 1914?

\section{INDEX}

Mots-clés : Géologie, géographie militaire, Grande Guerre, Epistémologie

Keywords : Geology, Military geography, Epistemology, Great War, France

\section{AUTEUR}

\section{PHILIPPE BOULANGER}

Professeur des universités en géographie à Sorbonne Université. Il est l'auteur, entre autres, de La France devant la conscription1914-1922 (Economica, 2001), La géographie militaire française 1871-1939, (Economica, 2002), Géographie militaire(Ellipses, coll. Carrefour, 2006), Géographie militaire et géostratégie, enjeux et crises du monde contemporain(2015, 2éd.). 